Neurosurg Focus 10 (3):Article 2, 2001 Click here to return to Table of Contents

\title{
Chordomas and chondrosarcomas of the skull base: results and complications of surgical management
}

\author{
Laligam N. Sekhar, M.D., F.A.C.S., Ramachandran Pranatartiharan, M.Ch., \\ Amitabha Chanda, M.Ch., and Donald C. Wright, M.D. \\ Department of Neurosurgery, The George Washington University Medical Center, Washington, DC; \\ Mid-Atlantic Brain and Spine Institutes, Annandale, Virginia
}

\begin{abstract}
Object. Chordomas and chondrosarcomas are rare and difficult to treat tumors for which the optimum treatment modality remains controversial. The aim of this study was to evaluate the surgery-related results and complications in a series of patients in whom radical resection was the treatment of choice.

Methods. The authors conducted a retrospective analysis of the surgery-related results and complications associated with chordoma and chondrosarcoma in 64 patients of whom $33(52 \%)$ had previously undergone some form of treatment. Total or near-total excision was achieved in $56 \%$ and this rate increased to $68 \%$ in patients without prior treatment. The main complications were postoperative cerebrospinal fluid leakage, intraoperative arterial injury, and new-onset cranial nerve deficits. Arterial injury occurred only and perioperative death occurred more often in patients who had undergone previous treatment.

Conclusions. Analysis of the results provides support for a policy of radical excision of chordomas and chondrosarcomas at the time of first presentation. A higher incidence of procedure-related complications is found in patients who have already undergone surgery and radiotherapy.
\end{abstract}

KEY WORDS • chordoma • chondrosarcma • skull base

Chordomas and chondrosarcomas are rare tumors, each comprising approximately $0.1 \%$ of all brain tumors. The majority of skull base chordomas are midline tumors whereas chondrosarcomas are paramedian with a predilection for the sphenopetroclival area. Because they share similar biological behavior, radiological features, and surgical treatment, they are addressed together in this paper. As they can be histologically similar, the distinction is usually based on immunochemical findings. Their location, propensity to infiltrate cancellous bone, and notorious ability to recur make them difficult tumors to treat. However, the use of skull base approaches has made possible an extensive resection of these tumors. Proton beam radiotherapy and radiosurgery are useful as adjuvant therapies but long-term results are available only for the former. ${ }^{1}$

Abbreviations used in this paper: $\mathrm{BA}=$ basilar artery; $\mathrm{CA}=$ carotid artery; $\mathrm{CSF}=$ cerebrospinal fluid; ICA = internal CA; $\mathrm{MR}=$ magnetic resonance.

\section{CLINICAL MATERIAL AND METHODS}

\section{Patient Population}

In this study we focus on the surgery-related results and complications in a series of 64 patients treated by the senior authors (L.N.S., D.C.W.) at the George Washington Medical Center and Inova Fairfax Hospital between 1993 and 2000. There were 42 chordomas and 22 chondrosarcomas. More than $50 \%$ of patients had previously undergone some form of treatment: surgery in 31 patients and radiotherapy in 15 .

\section{Preoperative Evaluation}

Magnetic resonance imaging was the main diagnostic modality used to delineate the extent of tumor and its margin, the relationship of tumor to the brainstem and cavernous sinus, and displacement or encasement of the ICA, $\mathrm{BA}$, and their branches. An angiogram was obtained in most patients to visualize the smaller vessels less reliably 
TABLE 1

Surgery-related complications and outcome stratified according to previous treatment $(s) *$

\begin{tabular}{lcccc}
\hline \hline & \multicolumn{4}{c}{ No. of Patients (\%) } \\
\cline { 2 - 5 } & $\begin{array}{c}\text { Chordoma } \\
\text { Factor }\end{array}$ & $\begin{array}{c}\text { Chondro- } \\
\text { sarcoma } \\
(22 \mathrm{pts})\end{array}$ & $\begin{array}{c}\text { No } \\
\text { Treatment } \\
(31 \mathrm{pts})\end{array}$ & $\begin{array}{c}\text { Prior } \\
\text { Treatment } \\
(33 \mathrm{pts})\end{array}$ \\
\hline tumor resection & & & \\
total & $25(59)$ & $11(50)$ & $21(68)$ & $15(46)$ \\
subtotal & $12(28)$ & $8(36)$ & $9(29)$ & $11(33)$ \\
partial & $5(11)$ & $3(14)$ & $1(3)$ & $7(21)$ \\
complications & $11(26)$ & $2(9)$ & $6(19)$ & $7(21)$ \\
CSF leak & $5(12)$ & $3(14)$ & $0(0)$ & $8(24)$ \\
vessel injury & & & & $7(21)$ \\
new CN defecit & $5(12)$ & $5(23)$ & $3(10)$ & $7(13)$ \\
$\quad$ 5th & $9(21)$ & $4(18)$ & $4(27)$ \\
$\quad$ 6th & $2(5)$ & $0(0)$ & $1(3)$ & $1(3)$ \\
major stroke & $5(12)$ & $0(0)$ & $1(3)$ & $4(12)$ \\
perioperative death & & &
\end{tabular}

$* \mathrm{CN}=$ cranial nerve; $\mathrm{pts}=$ patients.

demonstrated on MR imaging; to evaluate the collateral circulation, especially in cases of extensive tumors; and to evaluate the vein of Labbé as well as the size and pattern of flow of the sigmoid and transverse sinuses. A CA occlusion test was performed whenever the ICA was encased and narrowed or encased in a patient with a history of surgery or radiotherapy.

\section{Surgical Philosophy}

In patients who were medically fit and who had not undergone previous treatment we attempted complete tumor resection, in multiple stages if necessary. If a small tumor remnant remained after aggressive resection, proton beam radiotherapy was ususally inititated. In cases of previous radiotherapy, a more conservative, palliative resection was performed and followed by proton beam radiotherapy or radiosurgery depending on the size and location of remaining tumor. If any major arteries were damaged, they were repaired immediately.

\section{Surgical Approaches}

For extradural tumors the six main surgical approaches were the: 1) extended subfrontal; $;, 8$ 2) frontotemporal, orbitozygomatic, transcavernous; 3) subtemporal-infratemporal; $; 4$ ) extreme lateral transcondylar; ${ }^{3,7}$ 5) petrosal; ${ }^{6}$ and 6) the transoral transpharyngeal approach. ${ }^{2}$ For primarily intradural tumors, the approaches used were: 1) petrosal with partial labyrinthectomy and petrous apicectomy, ${ }^{6}$ and 2) frontotemporal, transcavernous.

\section{RESULTS}

\section{Outcome and Complications}

Total and near-total excision was achieved in 36 patients $(56.25 \%)$. In 20 patients $(31.25 \%)$ subtotal excision ( $>90 \%$ of tumor removed) was achieved, and in eight $(12.5 \%)$ partial excision was achieved. Of the 31 patients without prior treatment, total excision was achieved in $21(67.7 \%)$. Table 1 provides a summary of the results and complications according to tumor type and prior treat-

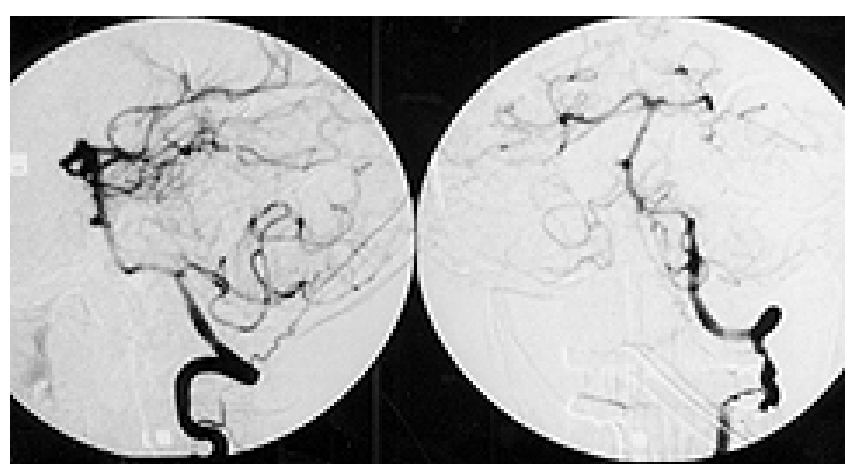

Fig. 1. Case 1. Vertebral artery angiograms, oblique (left) and anteroposterior (right) views demonstrating a mid-BA pseudoaneurysm.

ment received. Leakage of CSF occurred in 13 patients (20.3\%), of whom five developed meningitis and five patients required surgery to stop the CSF leak. Five patients (7.8\%) died within 3 months of surgery. All eight patients who sustained intraoperative vascular injury had previously undergone surgery and five had been irradiated. In all cases immediate vessel repair and/or bypass grafting was performed, but three patients died in the postoperative period. One previously untreated patient died of a delayed hemorrhage from a weakened BA, which showed microscopic invasion of tumor. The most common new permanent neurological deficit was sixth cranial nerve palsy, which occurred in 12 patients (18.7\%). This was followed by trigeminal nerve involvement in $(14 \%)$ nine patients. There was no statistical difference between the mean preoperative Karnofsky Performance Scale score (82.1) and the mean postoperative score (78.1) in survivors at 1 year.

\section{CASE ILLUSTRATIONS}

\section{Case 1}

This 62-year-old woman presented with gait ataxia. A large clival chordoma was diagnosed. A partial excision of tumor had been performed elsewhere via the transsphenoidal route. We performed a gross-total excision of the tumor via the petrosal approach with a partial labyrinthectomy and petrous apicectomy. During the resection bleeding occurred from a hole in the BA, which was sutured immediately. Postoperatively cognitive function was normal. An angiogram revealed midbasilar stenosis on the 1st postoperative day, which resolved by the 4th postoperative day. She developed a CSF leak, which was treated by transsphenoidal packing and a lumbar subarachnoid drain. On the 17th postoperative day the patient suffered major epistaxis, which ceased spontaneously. An angiogram revealed a pseudoaneurysm of the BA (Fig. 1). A leftsided external carotid-posterior cerebral artery saphenous vein bypass graft was performed, followed by trapping of the BA. However, this was unsuccessful with the patient dying due to a massive brainstem infarct and temporal lobe swelling.

\section{Case 2}

This 30-year-old man presented with headache and diplopia. He had undergone two attempts at transsphenoidal 


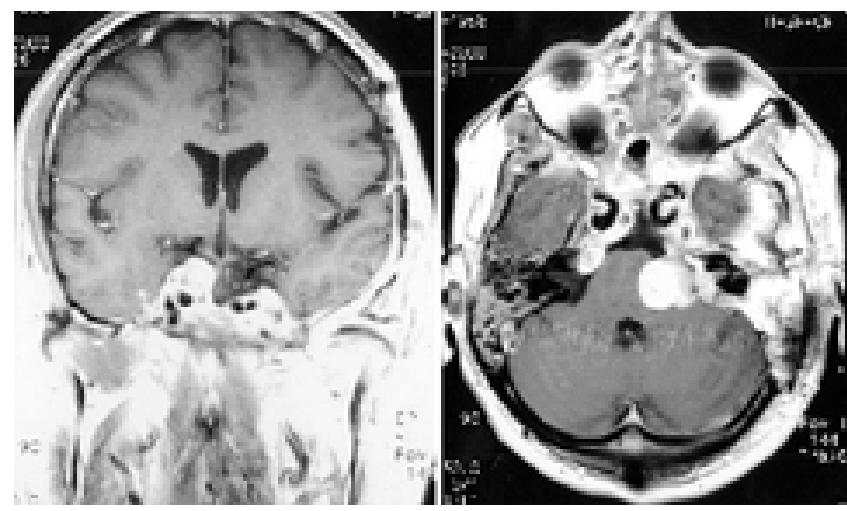

Fig. 2. Case 2. Magnetic resonance images. Left: Coronal image revealing tumor in the cavernous sinus on both sides. Right: Axial image revealing tumor in the left cerebellopontine angle and cavernous sinus on both sides.

excision elsewhere, the last one being abandoned due to sudden torrential bleeding. This had been followed by gamma knife surgery. We performed near-total resection of tumor, which led to complete recovery of vision in the left eye, but rapid regrowth of the tumor necessitated surgery within a year. A MR image revealed a tumor in the left petroclival region and both cavernous sinuses (Fig. 2). A frontotemporal craniotomy, orbital osteotomy, and partial excision of tumor were performed. Brisk arterial bleeding from the intracavernous CA that occurred while removing tumor from the right cavernous sinus was controlled by packing. A radial artery graft from the right cervical ICA to the right middle cerebral artery was done, and the remaining tumor in the cavernous sinus was removed. Postoperative angiography demonstrated a patent graft with good blood flow (Fig. 3). Further regrowth of tumor has occurred and the patient has required additional surgical intervention.

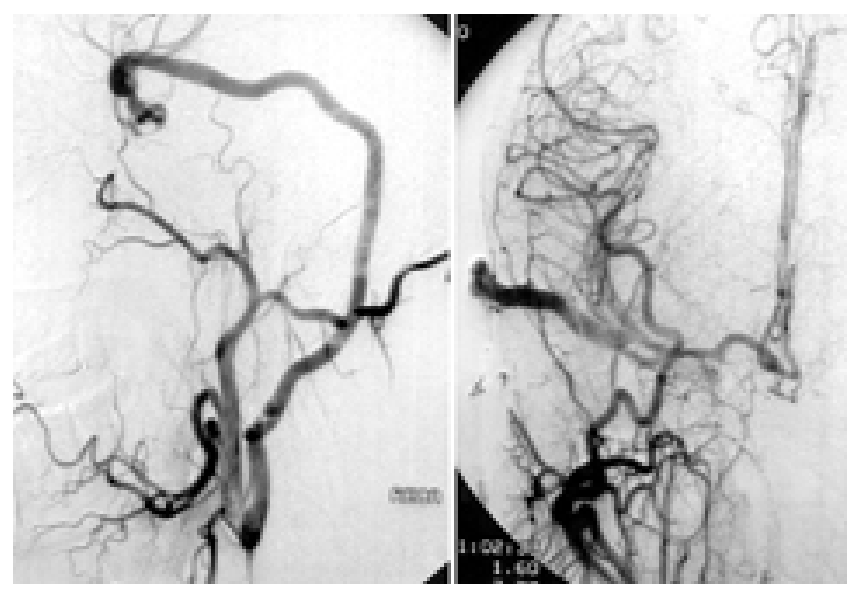

Fig. 3. Case 2. Right CA angiograms. Left: Lateral angiogram demonstrating the radial artery bypass graft from right ICA to right middle cerebral artery. Right: Anteroposterior angiogram demonstrating good filling of the middle cerebral artery and both anterior cerebral arteries from the graft.

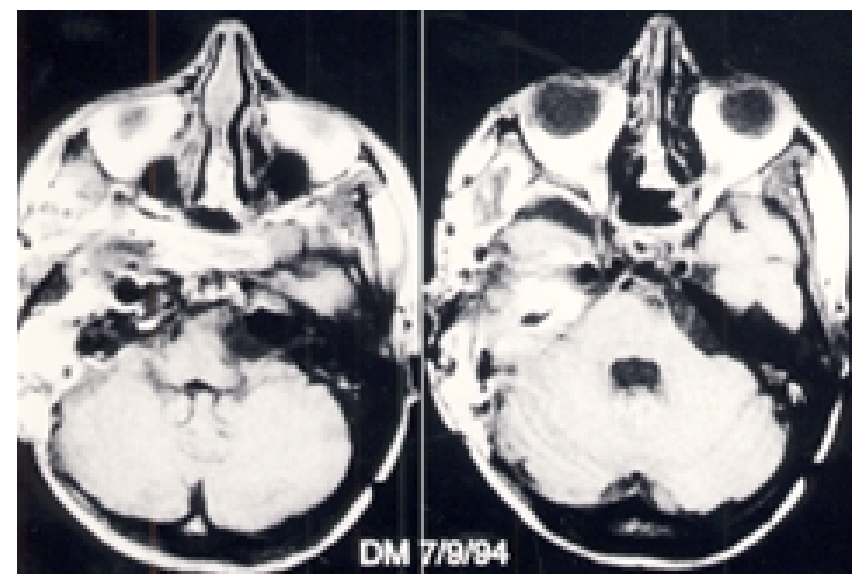

Fig. 4. Case 3. Axial $\mathrm{T}_{1}$-weighted MR images demonstrating the vascularized flap in the sphenoid sinus, petroclival area.

\section{Case 3}

This 33-year-old woman presented with recurrence of a sphenopetroclival chordoma after being treated with both surgery and irradiaion. She underwent a gross-total excision of tumor in two stages. A CSF leak developed after the first operation, and this subsided after placement of a lumbar subarachnoid drain. The CSF leak recurred after the second operation. A frontotemporal craniotomy and orbital osteotomy were performed, and the leak in the sphenoid sinus was repaired using fat and a vascularized flap (Fig. 4).

\section{DISCUSSION}

Cerebrospinal fluid leakage was the most common postoperative complication, occurring in $20 \%$ of patients. Meningitis occurred in five of these patients and resulted in pneumonia and gram-negative sepsis and death in one patient. Prevention of CSF leakage thus becomes an important strategy and is achieved by meticulous dural closure and reconstruction with free or vascularized graft (as in Case 3) and fat graft to obliterate dead space. Vascular injury was the main cause of mortality and major morbidity in this series (as in Case 1). All of these injuries occurred in patients who had previously undergone surgery or radiotherapy. Based on these results we have come to recommend only palliative resection in patients with a history of radiotherapy. Even so, a higher incidence of complications should be expected. We advocate radical excision in as many stages and with different approaches as required at the time of first presentation. Although the tendency of the tumor to recur makes reoperation necessary in some cases, every effort must be undertaken to avoid this by making the excision as radical as possible in the first surgical procedure. Patients with tumor remnants are advised to undergo proton beam radiotherapy or gamma knife surgery.

\section{References}

1. Hug EB, Loredo LN, Slater JD, et al: Proton radiation therapy 
for chordomas and chondrosarcomas of the skull base. J Neurosurg 91:432-439, 1999

2. Kingdom TT, Nockels RP, Kaplan MJ: Transoral-transpharyngeal approach to the craniocervical junction. Otolaryngol Head Neck Surg 113:393-400, 1995

3. Salas E, Sekhar LN, Ziyal IM, et al: Variations of the extremelateral craniocervical approach: anatomical study and clinical analysis of 69 patients. J Neurosurg 90 (Spine 4):206-219, 1999

4. Sekhar LN, Nanda A, Sen CN, et al: The extended frontal approach to the tumors of the anterior, middle, and posterior skull base. J Neurosurg 76:198-206, 1992

5. Sekhar LN, Salas EL: The subtemporal transzygomatic approach and the subtemporal infratemporal approach, in Sekhar LN, de Oliveira E (eds): Cranial Microsurgery: Approaches and Techniques. New York: Thieme, 1999, pp 413-431

6. Sekhar LN, Schessel DA, Bucur SD, et al: Partial labyrinthectomy petrous apicectomy approach to neoplastic and vascu- lar lesions of the petroclival area. Neurosurgery 44:537-552, 1999

7. Sekhar LN, Tzortzidis F, Salas EL: Extreme lateral retrocondylar and transcondylar approaches and combined approaches, in Sekhar LN, de Oliveira E (eds): Cranial Microsurgery: Approaches and Techniques. New York: Thieme, 1999, pp 464-481

8. Sekhar LN, Wright DC: Resection of anterior, middle, and posterior cranial base tumors via the extended subfrontal approach, in Sekhar LN, de Oliveira E (eds): Cranial Microsurgery: Approaches and Techniques. New York: Thieme, 1999, pp 82-90

Manuscript received January 15, 2001.

Accepted in final form February 9, 2001.

Address reprint requests to: Laligam N. Sekhar M.D., F.A.C.S., Mid-Atlantic Brain and Spine Institutes, 3301 Woodburn Road \#202, Annandale, Virginia 22003. 